\title{
RESULTS OF OPTICAL MONITORING OF THE DWARF NOVA SS CYG DURING THE 1993 CAMPAIGN OF CO- ORDINATED OBSERVATIONS WITH THE ASCA SATELLITE
}

\author{
IRINA VOLOSHINA \\ Sternberg Astronomical Institute, 119899 Moscow, Russia \\ e-mail:volosh@sai.msk.su
}

SS Cyg is the brightest known and therefore best studied classical dwarf nova and it was the first one from which $\mathrm{X}$-ray radiation was discovered. SS Cyg is unique because it has been detected at very wide range of energies, from a few eV up to $\sim 10 \mathrm{keV}$ (Jones \& Watson 1992). It was chosen as the first cataclysmic variable for $\mathrm{X}$-ray observations with the Japanese satellite ASCA. Simultaneous optical photometric and spectroscopic observations were also provided for completeness of the study.

SS Cyg was observed by ASCA in 1993 from 20:47 UT May 26 to 19:05 May 27. Our photometric monitoring of SS Cyg was started two days before, on May 24, and lasted two weeks. The observations were made with $60 \mathrm{~cm}$ telescope of the Sternberg Astronomical Institute in Crimea. The star BD $+42^{\circ} 4186$ served as the local standard. The measurement errors do not exceed $1 \%$. The results of our $U B V$ photometry are given in Table 1 . Using these data and the measurements communicated by the AFOEV the light curve of SS Cyg was constructed. The analysis of this light curve shows that the ASCA observations were made while the system was at outburst maximum and on the first steps of decline. The outburst started on May 17 and ended on June 3 when SS Cyg returned to quiescence. According to the criteria described in Howarth (1978), such as the form of the light curve at maximum, the magnitude at the maximum, the outburst width at $9.5 \mathrm{mag}$, the outburst width at $10.5 \mathrm{mag}$ and the rate of decline, we may conclude that this outburst is an anomalous one.

\section{References}

Howarth, I.D., 1978, J.British Astron.Assoc., 88, 458

Jones, M.H., Watson, M.G., 1992, MNRAS, 257, 633 
TABLE 1. Photoelectric Photometry of SS Cyg

\begin{tabular}{|c|c|c|c|c|}
\hline Date & $\mathrm{JD}_{\odot}$ & $V$ & $B-V$ & $U-B$ \\
\hline \multirow[t]{2}{*}{24 May } & 2449132.525 & $9^{m} .003$ & $-0^{m} .056$ & $-0^{m} .940$ \\
\hline & 529 & 8.998 & -0.052 & -0.940 \\
\hline \multirow[t]{2}{*}{25 May } & 2449133.478 & 9.070 & -0.005 & -0.855 \\
\hline & 482 & 9.039 & -0.057 & -0.846 \\
\hline \multirow[t]{6}{*}{26 May } & 2449134.417 & 9.345 & -0.047 & -0.916 \\
\hline & 422 & 9.390 & -0.004 & -0.931 \\
\hline & 428 & 9.376 & -0.012 & -0.946 \\
\hline & 433 & 9.390 & -0.028 & -0.950 \\
\hline & 439 & 9.402 & -0.014 & -0.964 \\
\hline & 448 & 9.543 & -0.019 & -0.969 \\
\hline \multirow[t]{2}{*}{$30 \mathrm{May}$} & 2449138.453 & 10.847 & -0.087 & -0.996 \\
\hline & 459 & 10.884 & -0.061 & -0.894 \\
\hline \multirow[t]{5}{*}{31 May } & 2449139.424 & 11.149 & 0.150 & -0.823 \\
\hline & 431 & 11.329 & 0.165 & -0.822 \\
\hline & 436 & 11.243 & 0.081 & -0.906 \\
\hline & 442 & 11.207 & 0.127 & -0.940 \\
\hline & 447 & 11.183 & 0.157 & -0.849 \\
\hline \multirow[t]{4}{*}{2 June } & 2449141.450 & 11.718 & 0.455 & -0.894 \\
\hline & 455 & 11.915 & 0.524 & -0.867 \\
\hline & 460 & 11.987 & 0.529 & -0.873 \\
\hline & 465 & 12.006 & 0.548 & -1.024 \\
\hline \multirow[t]{3}{*}{3 June } & 2449142.512 & 12.137 & 0.513 & -0.952 \\
\hline & 517 & 12.165 & 0.601 & -1.067 \\
\hline & 522 & 12.144 & 0.465 & -1.017 \\
\hline \multirow[t]{14}{*}{7 June } & 2449146.356 & 12.011 & 0.395 & -1.028 \\
\hline & 363 & 12.103 & 0.430 & -0.983 \\
\hline & 369 & 12.039 & 0.471 & -1.056 \\
\hline & 374 & 11.996 & 0.484 & -0.956 \\
\hline & 381 & 11.953 & 0.452 & -1.013 \\
\hline & 387 & 12.086 & 0.523 & -0.968 \\
\hline & 393 & 12.076 & 0.552 & -0.942 \\
\hline & 400 & 11.873 & 0.356 & -1.020 \\
\hline & 406 & 11.868 & 0.488 & -0.988 \\
\hline & 411 & 11.963 & 0.463 & -1.050 \\
\hline & 417 & 11.929 & 0.454 & -0.992 \\
\hline & 424 & 11.970 & 0.528 & -1.051 \\
\hline & 431 & 12.141 & 0.577 & -0.937 \\
\hline & 438 & 12.099 & 0.763 & -1.121 \\
\hline
\end{tabular}

